\title{
Minimal modules over serial rings
}

\author{
Sonia L'Innocente, Vera Puninskaya, Carlo Toffalori \\ Department of Mathematics and Computer Science \\ University of Camerino \\ Via Madonna delle Carceri 9, 62032 Camerino, Italy \\ \{sonia.linnocente, vera.puninskaya, carlo.toffalori\}@unicam.it
}

Keywords. Serial ring, uniserial ring, strongly minimal, pseudo-strongly minimal, quasi minimal, weakly minimal.

\begin{abstract}
We investigate several model theoretic minimalities in the framework of modules over a given serial ring $R$.
\end{abstract}

\section{Introduction}

Throughout this paper, ring will always mean an associative ring with identity, and module a right unital module. Rings are possibly uncountable (unless otherwise stated). We are interested in classifying strongly minimal, quasi minimal, pseudo-strongly minimal and weakly minimal modules over a serial ring. Modules satisfying one of these minimalities can be viewed as having low model theoretic complexity and in this sense are easier to approach.

For instance, for $R$ an arbitrary countable ring, an infinite $R$-module $M$ is strongly minimal (s. m. from now on) if and only if every proper pp-subgroup of $M$ is finite, and quasi minimal (q. m.) if and only if $M$ is uncountable and every proper pp-subgroup of $M$ is countable.

A common key property of s. m. and q. m. modules is the following.

Remark 1.1 ([7], [8], [14], [15]) Let $M$ be a s. m., or q. m. module over $R$. Then $A n n_{R}(M)=\{r \in R: M r=0\}$ is a completely prime ideal and $M$ is a divisible module over the quotient domain $R / A n n(M)$. In particular

( for every $r \in R$ either $M r=0$ or $M r=M$.

Introducing pseudo-strong minimality requires some more details and comments. Indeed this notion was defined in [3] over commutative rings $R$; in this setting an $R$-module $M$ is called pseudo-strongly minimal (p. s. m. from now on) if and only if $M$ is of infinite length but every proper pp-subgroup of $M$ is of finite length as a module over $R$. In fact, over a commutative $R$ pp-subgroups are also $R$-submodules; but this may fail over a non-commutative $R$. Accordingly the definition of pseudo-strong minimality had to be arranged 
in a suitable way to be extended over group rings $D G$ for $D$ a Dedekind domain and $G$ a finite possibly non-abelian group: this was done in [7].

A possible way of extending pseudo-strong minimality to modules over any ring $R$ is the following. For every $R$-module $M$, let $Z_{R}(M)$ denote the subring of $R$ of the elements $r$ of $R$ such that, for all $s \in R, r s$ and $s r$ act in the same way on $M$; in other words

$$
Z_{R}(M)=\{r \in R: \forall s \in R, M[r s-s r]=M\}
$$

(for $t \in R, M[t]$ denotes here the pp-subgroup of the elements of $M$ annihilated by $t$ ). Observe that $Z_{R}(M)$ extends the center of $R$ and that every pp-subgroup of $M$ is in a natural way a right module over $Z_{R}(M)$. Accordingly call $M$ pseudo-strongly minimal (p. s. m.) if and only if

- $M$ has infinite length over $R$ (and consequently over $Z_{R}(M)$ ),

- every proper pp-subgroup of $M$ over $R$ has finite length over $Z_{R}(M)$.

Note that this definition coincides with that of [3] over a commutative $R$ because under the commutativity assumption $Z_{R}(M)=R$ for every $M$. It also extends that over group rings $R=D G$ for $D$ a Dedekind domain and $G$ a finite group, so pseudo-strong minimality as defined in [7]; in fact, for every module $N$ over $Z_{R}(M) N$ has finite length over $Z_{R}(M)$ if and only if $N$ has finite length over $D$ because $Z_{R}(M)$ includes $D$ and has finite length over $D$.

Observe that a module $M$ p. s. m. over an arbitrary ring $R$ is p. s. m. even over $Z_{R}(M)$ because $Z_{Z_{R}(M)}(M)=Z_{R}(M)$.

This definition of pseudo-strong minimality preserves the basic properties observed in [3] and [7] in the particular cases of commutative rings and group rings. For instance, it turns out that:

- A p. s. m. $R$-module is totally transcendental (that is, it satisfies the descending chain condition on pp-subgroups).

- A p. s. m. $R$-module $M$ is connected (in fact, as $M$ is totally transcendental, it makes sense to consider the connected component $M^{0}$ of $M$, as a minimal pp-subgroup of finite index; if $M^{0} \neq M$, then $M^{0}$ has finite length over $Z_{R}(M)$, which implies that $M$ has finite length over $Z_{R}(M)$, too).

More notably, a p. s. m. $R$-module $M$ shares with s. m. and q. m. modules the key property in Remark 1.1. In particular

( $\quad$ for every $r$ in $R$, either $M r=0$ or $M r=M$.

To check this, consider for every $r \in R$

$$
0 \rightarrow M[r] \rightarrow M \rightarrow M r \rightarrow 0
$$


as an exact sequence over $Z_{R}(M)$. Observe that, if $M r$ is neither $M$ nor 0 , then both $M r$ and $M[r]$ are proper subgroups of $M$, and so both $M r$ and $M[r]$ have finite length over $Z_{R}(M)$, which implies that $M$ is of finite length, too.

Another basic fact concerning $Z_{R}$ is the following one.

Remark 1.2 If $M=M_{0} \oplus M_{1}$, then $Z_{R}(M)=Z_{R}\left(M_{0}\right) \cap Z_{R}\left(M_{1}\right)$. Hence, if $\varphi(v)$ is a pp-formula and $\varphi(M)$ is of finite length over $Z_{R}(M)$, then for each $i=0,1 \varphi\left(M_{i}\right)$ is of finite length over $Z_{R}(M)$ and over $Z_{R}\left(M_{i}\right)$.

Even weak minimality deserves a specific attention. Weakly minimal modules are those of $U$-rank 1 , but can be equivalently introduced in the following way: A module $M$ over $R$ is weakly minimal (w. m.) if and only if every pp-subgroup of $M$ is either finite or of finite index. Hence w. m. modules may fail to satisfy $(\star)$, although they admit the following related and weaker property: For every $r \in R$, either $M r$ is finite, or $M r$ has finite index and $M[r]$ is finite (see [9] and $[10])$.

With this in mind, we are going to examine the previous four minimalities (s. m., q. m., p. s. m. and w. m.) within modules over a serial ring $R$. Recall that an $R$-module $M$ is said to be uniserial if the lattice of submodules of $M$ is a chain and serial if $M$ is a direct sum of uniserial modules; a ring $R$ is said to be uniserial (serial) if both $R_{R}$ and ${ }_{R} R$ are uniserial (serial) modules.

Here is the plan of the paper. First we see in $\S 2$ that $(\star)$ easily reduces our analysis from serial to uniserial rings. At this point some previous results of the second author ([14] and [15]) apply and solve the s. m. case in a satisfactory way. Even the q. m. case can be treated on the basis of what is shown in [3]. So, when $(\star)$ holds, it remains to examine p. s. m. modules over uniserial rings: this will be the content of $\S 3$. As said, w. m. modules require an ad-hoc approach because they may not satisfy $(\star) ; \S 4$ will be devoted to them, in particular we will see that even in their setting most of the analysis concerns the uniserial case.

The main part of the paper is that regarding the pseudo-strong case. The other minimalities are either already treated in the literature, although sometimes implicitly, as in the q. m. case, or too general to allow a sharp description; this is what happens, for instance, in the w. m. case. Anyhow we think it useful to provide a general outline of all of them in the serial framework.

We refer to [2] and [17] for (modules over) serial rings, to [11] for noncommutative rings and to [5], [13] and [19] for model theory of modules. Our notation will be standard.

We thank our anonymous referee for carefully reading our original manuscript and suggesting several simplifications. 


\section{From serial to uniserial, and from strong to quasi}

When $(\star)$ holds, our analysis quickly reduces from serial rings to uniserial domains.

Lemma 2.1 Let $R$ be a serial ring, $M$ be a nonzero module over $R$ satisfying $(\star)$. Then $R / \operatorname{Ann}_{R}(M)$ is a uniserial domain.

Proof. ( $\star$ ) implies that $R / A n n_{R}(M)$ is a domain. But $R / A n n_{R}(M)$ is also serial, as a homomorphic image of a serial ring. Hence $R / A n n_{R}(M)$ is uniserial. $\dashv$

Accordingly every s. m., or q. m., or p. s. m. module $M$ over a serial ring $R$ is in fact a module over the uniserial domain $R / A n n_{R}(M)$, and as such it remains s. m., q. m., p. s. m. respectively. In particular, regarding pseudo-strong minimality, observe that $Z_{R}(M)$ includes $A n n_{R}(M)$ and the corresponding quotient ring $Z_{R}(M) / A n n_{R}(M)$ is just $Z_{R / A n n_{R}(M)}(M)$ because the natural projection of $R$ onto $R / A n n_{R}(M)$ clearly sends elements of $Z_{R}(M)$ into elements of $Z_{R / A n n_{R}(M)}(M)$ and, conversely, every element of $Z_{R / A n n_{R}(M)}(M)$ can be reached in this way by some element in $Z_{R}(M)$. Then we can reduce our analysis to the case of faithful - indeed divisible - modules over uniserial domains. On the other hand, s. m. modules over right distributive rings, and hence in particular over uniserial rings $R$, were completely described in [14] (see also [15]). Let us recall the key result in [14] in the case of uniserial rings. $\operatorname{Jac}(R)$ denotes here, as usual, the Jacobson radical of $R$.

Theorem 2.2 [14] Let $M$ be a faithful s. m. indecomposable module over a uniserial ring $R$. Then $R$ is a domain and either

1. $M$ is torsionfree over $R$ and then $M$ is isomorphic to the quotient (skew) field $Q(R)$ of the uniserial domain $R$, or

2. $M$ has $R$-torsion and then $R$ is a noetherian uniserial domain, $R / \operatorname{Jac}(R)$ is a finite field and $M$ is isomorphic to the injective envelope of the unique simple module $R / J a c(R)$.

The proof refers in a crucial way to results in [18]. Moreover the converse is also true ([14]): every module satisfying 1 ) or 2) is strongly minimal. On this basis, one obtains the following classification of s. m. modules over uniserial rings.

Theorem 2.3 Let $R$ be a uniserial ring. An infinite module $M$ over $R$ is s. $m$. if and only if one of the following holds.

1. $M$ decomposes as

$$
\left.Q\left(R^{\prime}\right)^{(\alpha)} \oplus \bigoplus_{P \in S}\left(E_{R^{\prime}}\left(R^{\prime} / P\right)\right)^{\left(\beta_{P}\right.}\right)
$$


where $\alpha$ and the $\beta_{P}$ are cardinals and the $\beta_{P}$ are finite; $R^{\prime}=R / J$ for some completely prime ideal of $R$ such that $R / J$ is not a skew field; $Q\left(R^{\prime}\right)$ is a classical right quotient ring of $R^{\prime} ; S$ is a set of completely prime ideals of $R^{\prime}$ such that $R^{\prime} / P$ is a finite field, the right localization $R_{(P)}^{\prime}$ of $R^{\prime}$ at $P$ is right noetherian and its ideals are

$$
p R_{(P)}^{\prime} \supset p^{2} R_{(P)}^{\prime} \supset \ldots \supset p^{n} R_{(P)}^{\prime} \supset \ldots
$$

where $p$ is a generator of the largest ideal in $R_{(P)}^{\prime}$; finally, every nonzero element $r \in R^{\prime}$ is contained in at most finitely many ideals of $S$.

2. $M \simeq(R / I)^{(\gamma)}$ where $I$ is a completely prime ideal of $R$ and $R / I$ is a skew field.

In this way the s. m. analysis is accomplished even over a serial ring $R$. Indeed a sharper description of strong minimality can be obtained under stronger assumptions on $R$, for instance when $R$ is a serial indecomposable basic ring.

In fact, let $e_{1}, \ldots, e_{n}$ be a complete system of pairwise orthogonal indecomposable idempotents of $R$. Define $R_{i}=e_{i} R e_{i}, i=1, \ldots, n$ and $R_{i j}=e_{i} R e_{j}$, $i, j=1, \ldots, n ; i \neq j$. Then the $R_{i}$ are uniserial rings and the $R_{i j}$ are $\left(R_{i}, R_{j}\right)$ bimodules. Recall that $R$ is said to be basic if all modules $e_{i} R, i=1, \ldots, n$, are pairwise nonisomorphic. If $i \neq j$ then, since $R$ is a basic ring, we have that $R_{i j} R_{j i}$ is a proper ideal of the ring $R_{i}$. By [12] this ideal is completely prime if $R_{i j} \neq 0$ or $R_{j i} \neq 0$. Since $R$ is indecomposable then for every $i$ there is $k$ such that $R_{i k} \neq 0$ or $R_{k i} \neq 0$. Therefore the ideal $P_{i}$ generated by the set $\left\{e_{j} \mid j \neq i\right\}$ is a proper ideal (since $e_{i} \notin P_{i}$ ) and $P_{i}^{\prime}=e_{i} P e_{i}$ is a completely prime ideal of the form $R_{i k} R_{k i}$ for some $k$. From this it follows that $P_{i}$ is a Goldie prime ideal. Notice also that if the ideal $\operatorname{Jac}\left(R_{i}\right)$ is non-nilpotent then the ideal $J a c^{\omega}\left(R_{i}\right)=\cap_{n} J a c\left(R_{i}\right)^{n}$ is completely prime.

Let $M$ be a s. m. $R$-module, set for simplicity $P=A n n_{R}(M)$. Then $R / P$ is a uniserial domain and $P$ contains all but one of the orthogonal indecomposable idempotents $e_{1}, \ldots, e_{n}$. Suppose that $P$ does not contain $e_{i}$; then $P$ includes $P_{i}$. Therefore $P$ is a Goldie prime ideal containing some (unique) ideal $P_{i}$. If $M$ has $R$-torsion then $R / P$ is a noetherian uniserial domain. Therefore $\operatorname{Jac}\left(R_{i}\right)$ is a nonnilpotent ideal and $J a c^{\omega}\left(R_{i}\right)=e_{i} P e_{i}$. In conclusion, combining this and Theorem 2.2, we have the following.

Theorem 2.4 Let $M$ be a s. m. indecomposable module over a serial indecomposable basic ring $R$, let $e_{1}, \ldots, e_{n}$ be a complete set of pairwise orthogonal indecomposable idempotents of $R$ and for every $i=1, \ldots, n$ let $P_{i}$ denote the ideal generated by the $e_{j}$ for $j \neq i$. Then there is a Goldie prime ideal $P$ containing some (unique) ideal $P_{i}$ such that either

1. $M$ is the quotient (skew) field of the uniserial domain $R / P$, or

2. $J a c\left(R_{i}\right)$ is a non-nilpotent ideal, $J_{a c}^{\omega}\left(R_{i}\right)=e_{i} P e_{i}, S_{i}=R_{i} / J a c\left(R_{i}\right)$ is a finite field and $M$ is the injective envelope over the ring $R / P$ of the unique simple module $S_{i}$. 
By Theorem 2.2 the converse is also true: every module satisfying the property 1 ) or the property 2 ) is strongly minimal.

Lemma 2.1 applies also to the q. m. case and reduces its analysis from serial to uniserial rings. Moreover uniserial domains are Ore and, although quasi minimality is not preserved under elementary equivalence, it is known that, over a countable Ore domain, a module is elementarily equivalent to some $\mathrm{q}$. $\mathrm{m}$. module if and only if it is divisible: this fact is observed in [8], but is essentially proved in [4]. This changes in some sense our perspective, because our investigation of q. m. modules over uniserial domains becomes a part of the characterization of divisible modules over Ore domains. The latter purpose can be developed on the basis of the corresponding investigation over commutative domains in [3]. [16] gives the details of this analysis, but it turns out that the main results and proofs are basically the same as in [3]. So we omit here a detailed report.

At this point we have to examine the p. s. m. case over uniserial domains, and we will devote the next section to this matter.

\section{$3 \quad$ Pseudo-strong minimality}

As said, we deal here with p. s. m. modules $M$ over a uniserial domain $R$. We refer to the parallel analysis of the s. m. case in [15], § 4.3 .

Remarks 3.1 1. First of all it is worth underlining that, just due to uniseriality, for all $t, t^{\prime} \in R$, either $t R \subseteq t^{\prime} R$ or, conversely, $t^{\prime} R \subseteq t R$, in other words either $t=t^{\prime} s$ for some $s \in R$ or $t^{\prime}=t s$ for some $s \in R$.

2. As already illustrated in the previous sections, we can assume that $M$ is divisible.

3. Now recall the result in [1], Corollary 1.6, and [17], Lemma 11.1, saying that, over a serial ring $R$, every pp-formula is equivalent to a finite sum of conjunctions of divisibility formulas $s \mid v$ or torsion formulas $v t=0$ where $s \in e R, t \in R f$ and $e, f$ are in a complete system of pairwise orthogonal idempotents. Over a uniserial $R$, we can assume that this finite sum just consists of formulas $s \mid v \wedge v t=0$ with $s$ and $t$ in $R$. Within divisible modules, any divisibility condition like $s \mid v$ with $s \neq 0$ trivially holds true, so we can reduce any finite sum as before to a torsion statement like $\sum_{t} v t=0$. Again using uniseriality and the previous remark 1, we easily deduce that each pp-formula over a uniserial domain $R$ is equivalent within divisible $R$-modules $M$ to a single torsion condition $v t=0$ with $t \in R$, which emphasizes the key role of annihilator pp-subgroups $M[t]=$ $\{m \in M: m t=0\}$ in our setting.

For every (divisible) $R$-module $M$ let us introduce $P_{R}(M)$ as the set of the elements $t \in R$ such that $M[t] \neq 0$, hence some nonzero $m \in M$ is annihilated by $t$. 
Lemma 3.2 Let $M$ be a divisible module over a uniserial domain $R$. Then $P_{R}(M)$ is a completely prime ideal of $R$.

Proof. This is a general fact and does not depend on pseudo-strong minimality. Here are some details.

- The fact that $P_{R}(M)$ is closed under addition follows directly from the uniseriality assumption on $R$. To see this, take $t, t^{\prime}$ in $P_{R}(M)$, have to check that $t+t^{\prime}$ is in $P_{R}(M)$, too. For if $t, t^{\prime}$ are in $P_{R}(M)$ then by uniseriality we can assume that $t=t^{\prime} s$ for some $s$. Consequently, for any $m \in M$, if $t^{\prime}$ annihilates $m$, then $t$ does. In particular if some $m \neq 0$ satisfies $m t^{\prime}=0$, then $m t=0$ as well, and $m\left(t+t^{\prime}\right)=0$.

- The fact that $P_{R}(M)$ is closed under left and right multiplication by elements of $R$ follows from the divisibility assumption on $M$. To see this take $t \in P_{R}(M)$ and $r \in R$. We have to check that both $t r$ and $r t$ are in $P_{R}(M)$. This is trivial when $r$ is to the right or when $r=0$. So assume $r \neq 0$ and look at $r t$. Pick $m \in M$ such that $m \neq 0$ and $m t=0$. Divisibility provides $m^{\prime} \in M$ such that $m^{\prime} r=m$, whence $m^{\prime} r t=0$. But $m^{\prime} \neq 0$ and so $r t \in P_{R}(M)$ as expected.

- The rest of the proof follows directly from the definition of $P_{R}(M)$.

As a consequence, if $M$ is a divisible $R$-module, then the right localization $R_{\left(P_{R}(M)\right)}$ exists. Let $\bar{R}$ denote this localization below.

Lemma 3.3 Let $M$ be a divisible p. s. $m$. (indeed totally transcendental) module over a uniserial domain $R$. Then $M$ is $\Sigma$-injective.

Proof. As noted before, a p. s. m. $M$ is totally transcendental and consequently $\Sigma$-pure injective. A pure injective module is injective if and only if it is $\aleph_{0}$-injective, that is, for every $n \geq 2$, every system of fewer than $n R$-linear equations in one unknown $v$ that has a solution in some extension of $M$ has a solution in $M$. Therefore it suffices to show that $M$ is $\aleph_{0}$-injective. We prove this by induction on $n \geq 2$; the case $n=2$ (that is, one linear equation) is just the divisibility of $M$. So let us assume our claim true for $n$ and prove it for $n+1$. Suppose that the equations $v r_{i}=m_{i}\left(1 \leq i \leq n, m_{i} \in M\right.$ and $r_{i} \in R$ for every $i$ ) have a common solution $a$ in some extension $N$ of $M$. Using the uniseriality of $R$ and possibly rearranging the order of these equations we can assume $r_{n}=r_{1} t$ for some $t \in R$. Hence $m_{n}=a r_{n}=a r_{1} t=m_{1} t$. By induction there is some $b \in M$ satisfying $b r_{i}=m_{i}$ for $1 \leq i<n$. But then $b r_{n}=b r_{1} t=m_{1} t=m_{n} . \dashv$

In view of [18], Theorem 4.2, if $M$ is an indecomposable $\Sigma$-injective module over a uniserial ring (indeed over a right distributive ring) $R$, then $P_{R}(M)$ is a completely prime ideal, the right localization $\bar{R}=R_{\left(P_{R}(M)\right)}$ of $R$ exists and enjoys several nice properties (see Theorem 3.6 below). 
Let us point out now a couple of simple facts about $\bar{R}$. In the following lemmas we assume that $M$ is any module over $R$ such that $P_{R}(M)$ is a completely prime ideal. Note that any element of $R-P_{R}(M)$ acts invertibly on $M$.

Lemma $3.4 Z_{R}(M) \subseteq Z_{\bar{R}}(M)$.

Proof. Let $r \in R$ satisfy $m r s=m s r$ for every $m \in M$ and $s \in R$. For $s \in R-P_{R}(M)$, let $\bar{m}=m s^{-1}$ be the element of $M$ satisfying $\bar{m} s=m$. Then $m r=\bar{m} s r=\bar{m} r s$, whence $m r s^{-1}=\bar{m} r=m s^{-1} r$. Consequently for $t \in R$, $s \in R-P_{R}(M)$ and $m \in M m r t s^{-1}=m t s^{-1} r . \dashv$

Note that $Z_{\bar{R}}(M)$ also includes the elements $r^{-1}$ with $r \in Z_{R}(M)-P_{R}(M)$.

Lemma 3.5 Every pp-subgroup of $M$ over $\bar{R}$ is also a pp-subgroup over $R$.

Proof. Every atomic formula $v r s^{-1}=w$ with $r, s \in R$ and $s \notin P_{R}(M)$ is equivalent in $M$ to $v r=w s$. In this way every pp-formula $\varphi(v)$ over $\bar{R}$ can be translated into a pp-formula $\varphi^{\prime}(v)$ for which $\varphi(M)=\varphi^{\prime}(M)$.

In particular, for every $r=t s^{-1} \in \bar{R}, M[r]=M[t]$ and hence $M[r]$ is a ppsubgroup of $M$ over $R$.

Let us come back to the analysis of p. s. m. modules. We distinguish two cases, according to whether $P_{R}(M)$ is 0 or not.

Theorem 3.6 Let $M$ be a divisible module over a uniserial domain $R$. Suppose that $P_{R}(M) \neq 0$. Then the following conditions are equivalent:

1. $M$ is indecomposable and p. s. m.;

2. $M$ is canonically a module over $\bar{R}$ and indeed $M_{\bar{R}} \simeq E_{\bar{R}}(\bar{R} / \bar{P})$, where $E$ denotes injective hull, $\bar{R}$ is right noetherian, $\bar{P}=P_{R}(M)_{\left(P_{R}(M)\right)}$ is the largest ideal of $\bar{R}, \bar{R} / \bar{P}$ has finite length over $Z_{\bar{R}}\left(E_{\bar{R}}(\bar{R} / \bar{P})\right)$ and, if $p$ is a generator of $\bar{P}$, then the ideals of $\bar{R}$ are just $p \bar{R} \supset p^{2} \bar{R} \supset \ldots \supset p^{h} \bar{R} \supset$ $\ldots \supset 0$ (for $h$ a nonnegative integer).

Proof. $1 \Rightarrow 2$. Recall that $M$, as a p. s. m. module, is totally transcendental. As $M$ is indecomposable and $\Sigma$-injective, Theorem 4.2 of [18] applies and confirms that the right localization $\bar{R}$ of $R$ at the completely prime ideal $P_{R}(M)$ exists. It moreover ensures some further properties: $M$ is, as claimed, a faithful $\Sigma$-injective module over $\bar{R}$, and indeed $M$ is over $\bar{R}$ isomorphic to $E_{\bar{R}}(\bar{R} / \bar{P})$. Also, $\bar{R}$ is right noetherian and right uniserial, hence right duo (see [18] and [15], $\S 4.3)$. Finally, $\bar{P}$ is the largest ideal of $\bar{R}$.

Now let us examine the ideals of $\bar{R}$. First observe that, as $R$ is right duo, ideals just coincide with right ideals in $\bar{R}$. Every right noetherian uniserial domain is a principal right ideal domain; hence every ideal of $\bar{R}$ is of the form $t \bar{R}$ for some $t$. In particular let $p$ denote a generator of $\bar{P}$. Take two nonzero proper ideals $I=t \bar{R}$ and $I^{\prime}=t^{\prime} \bar{R}$ of $\bar{R}$. Observe that $M[I]=M[t]$ (and $M\left[I^{\prime}\right]=M\left[t^{\prime}\right]$ ). Due to (right) uniseriality, either $I \subseteq I^{\prime}$ or $I^{\prime} \subseteq I^{\prime}$. Assume for simplicity the former 
condition, so $t=t^{\prime} s$ for some $s \neq 0$ and conclude that $M\left[t^{\prime}\right] \subseteq M[t]$. Furthermore $I \neq I^{\prime}$ implies $M\left[t^{\prime}\right] \neq M[t]$ : take a nonzero $m \in M$ for which $m s=0$ and $m^{\prime} \in M$ such that $m^{\prime} t^{\prime}=m$, then $m^{\prime} t^{\prime} \neq 0$ and $m^{\prime} t=m^{\prime} t^{\prime} s=m s=0$. Now recall that $M$, as a totally transcendental $R$-module, satisfies the descending chain condition on pp-subgroups over $R$, in particular on annihilator subgroups $M[t]$ over $R$. Correspondingly, for every proper nonzero ideal $I$ of $R$, there are at most finitely many ideals $I^{\prime}$ of $\bar{R}$ containing $I$. This implies $\cap_{h} p^{h} \bar{R}=0$ and hence, as $\bar{R}$ is local, the ideals of $\bar{R}$ are just as said in Statement 2 of the theorem.

It remains to show the claim about the length of $\bar{R} / \bar{P}$. Put

$$
U=\left\{m \in M: A n n_{\bar{R}}(m) \subseteq \bar{P}\right\}=\bigcap_{t \in \bar{P}} M[t] .
$$

Then $U=M[p]$ and we can find a nonzero $r \in R$ for which $U=M[r]$. Due to the structure of ideals of $\bar{R}, U$ is a submodule of $M$ over $\bar{R}$, and the same holds true for $M\left[p^{n}\right]$ for every nonnegative integer $n$. In fact, take $r \in \bar{R}$ and $m \in M$ satisfying $m p^{n}=0$, note that $r p^{n}$ is in $p^{n} R$ because the latter is a two-sided ideal, hence $r p^{n}=p^{n} r^{\prime}$ for some $r^{\prime} \in R$, and conclude that $m r p^{n}=m p^{n} r^{\prime}=0$. By [18], Theorem 4.2, $U$ is a 1-dimensional vector space over the skew field $\bar{R} / \bar{P}$. Furthermore $U$ - as $M[r]$ - is a pp-subgroup of $M$ over $R$, and indeed a proper pp-subgroup because $r \neq 0$. Then pseudo-strong minimality implies that $U$ has finite length over $Z_{R}(M) . \bar{R} / \bar{P}$ is isomorphic to $U$ as a vector space over $\bar{R} / \bar{P}$, hence over $\bar{R}$ and a fortiori over $R$, which embeds into $\bar{R}$, and even over $Z_{R}(M)$. Hence $\bar{R} / \bar{P}$ has finite length over $Z_{R}(M)$, and so over $Z_{\bar{R}}(M)$ because the latter clearly extends $Z_{R}(M)$. As $M \simeq E_{\bar{R}}(\bar{R} / \bar{P}), U$ is of finite length over $Z_{\bar{R}}\left(E_{\bar{R}}(\bar{R} / \bar{P})\right)$

$2 \Rightarrow 1$. As $M$ is divisible, $P_{R}(M)$ is completely prime and hence the localization $\bar{R}$ exists. Furthermore $\bar{R}$ is local and right uniserial. Also, $\bar{P}=P_{R}(M)_{\left(P_{R}(M)\right)}$ is the maximal ideal of $\bar{R}$, and $M=E_{\bar{R}}(\bar{R} / \bar{P})$ is indecomposable (see [18], $\S \S$ 3 -4). Furthermore $M$ is $\Sigma$-injective and $\bar{P}=P_{\bar{R}}(M)$. At this point we need show that $M$ is p. s. m. over $R$.

It is easy to see that $M$ has infinite length over $R$. For, fix a generator $p$ of $\bar{P}$ and look at the ascending chain of subgroups $M\left[p^{n}\right]$ where $n$ is a positive integer. As said before, $M\left[p^{n}\right]$ is actually an $R$-submodule.

So it remains to prove that every proper $p p$-subgroup of $M$ over $R$ has finite length over $Z_{R}(M)$. So take a subgroup $M[r]$ with $r \in R, r \neq 0 ; M\left[r^{n}\right]$ can be also represented as $M\left[p^{n}\right]$ for some suitable nonnegative integer $n$. Incidentally notice that this implies that the pp-subgroups of $M$ over $R$ satisfy the descending chain condition and consequently that $M$ is totally transcendental. Consider any proper nonzero $Z_{R}(M)$-submodule $U$ of $M$, and look at $A n n_{\bar{R}}(U)$, so at the set of the elements $r \in \bar{R}$ such that $u r=0$ for every $u \in U$; this is a right ideal of $\bar{R}$, and so a two-sided ideal because $R$ - as a right uniserial and right noetherian ring - is right duo. Then $A n n_{\bar{R}}(M)$ equals $p^{n} \bar{R}$ for some $n$ (and 
hence is properly included in $\left.p^{n-1} \bar{R}\right)$. It follows that $M\left[p^{n-1}\right] \subset U \subseteq M\left[p^{n}\right]$. Consider the map from $M\left[p^{n}\right]$ to $M[p]$ sending any $a \in M\left[p^{n}\right]$ to $a p^{n-1}$. This is a $Z_{R}(M)$-module homomorphism because $Z_{R}(M) \subseteq Z_{\bar{R}}(M)$ and hence, for every $r \in Z_{R}(M) \subseteq Z_{\bar{R}}(M), a \in M\left[p^{n}\right]$ and $a r p^{n-1}=a p^{n-1} r$. Furthermore this map is onto because $M$ is divisible even over $\bar{R}$, and its kernel is $M\left[p^{n-1}\right]$. Consequently $M\left[p^{n}\right] / M\left[p^{n-1}\right] \simeq M[p]$ over $Z_{R}(M)$. Hence it suffices for our purposes to show that $M[p]$ has finite length over $Z_{R}(M)$, and this is true because $M[p]$ is a one-dimensional vector space over $\bar{R} / \bar{P}$ and $\bar{R} / \bar{P}$ has finite length over $Z_{\bar{R}}(M)=Z_{\bar{R}}\left(E_{\bar{R}}(\bar{R} / \bar{P})\right)$. $\dashv$

Note that the proof of the implication $1 \Rightarrow 2$ does not need the assumption that $M$ is of infinite length. In fact the only point where pseudo-strong minimality is used is to deduce that $M$ is totally transcendental. So the proof works even under the assumption of total transcendence (in addition to the hypothesis on $R$ and the fact that $M$ is indecomposable and the pp-subgroups of $M$ over $R$ have finite length over $Z_{R}(M)$ ).

The previous theorem illustrates what happens when $P_{R}(M) \neq 0$. The opposite case $P_{R}(M)=0$ (for $R$ a right uniserial domain and $M$ a divisible module over $R$ ) is easy to treat. In fact $M$ is even torsionfree over $R$. Also, $R$ is a (right) Ore domain, which implies that there exists a classical right ring of quotients $Q(R) ; M$ can be regarded as a vector space over $Q(R)$, hence $M$ is p. s. $\mathrm{m}$. exactly when it has infinite length over $R$.

The previous considerations lead to the following:

Theorem 3.7 Let $R$ be a right uniserial domain and $M$ be a divisible module over $R$. Then $M$ is $p$. s. $m$. if and only if either

1. $R$ is not a skew field and $M \simeq Q(R)^{(\alpha)} \oplus \oplus_{P \in I} E_{R_{(P)}}\left(R_{(P)} / P_{(P)}\right)^{\left(\beta_{P}\right)}$ where $Q(R)$ is a classical right ring of quotients of $R$; $I$ is a set of completely prime ideals of $R$ such that, for every $P \in I, \beta_{P}$ is finite, the right localization $R_{(P)}$ (exists and) is a right noetherian ring and the ideals of $R_{(P)}$ are just those of the form $p^{n} \bar{R}_{(P)}$ for $p$ a generator of $P_{(P)}$ and $R_{(P)} / P_{(P)}$ has finite length over $Z_{R_{(P)}}\left(E_{R_{(P)}}\left(R_{(P)} / P_{(P)}\right)\right) ; Q(R)^{(\alpha)}$ has infinite length over $R$ if $\beta_{P}=0$ for ever $P \in I$; finally, every nonzero $r \in R$ is contained in at most finitely many $P \in I$, or

2. $R$ is a skew field and $M \simeq R^{(\gamma)}$ for some infinite cardinal $\gamma$.

Proof. First suppose that $M$ is p. s. m. over $R$. If $R$ is a skew field, then $M$ is $R^{(\gamma)}$ for some cardinal $\gamma$, and pseudo-strong minimality forces $\gamma$ to be infinite. So suppose that $R$ is not a skew field. Due to pseudo-strong minimality, $M$ is totally transcendental and consequently can be represented over $R$ as a direct sum $M=\oplus_{j \in J} M_{j}^{\left(\beta_{j}\right)}$ of indecomposable pairwise non-isomorphic summands $M_{j}$. Note that each $M_{j}$ is either p. s. m. or of finite length over $R$ (by Remark 1.2). But even in the latter case $M_{j}$ is divisible and totally transcendental, and every pp-subgroup of $M_{j}$ is of finite length over $Z_{R}\left(M_{j}\right)$. When $P_{R}\left(M_{j}\right)=$ 
$0, M_{j}$ is just a vector space over $Q(R)$. So assume that $P_{R}\left(M_{j}\right)$ is not 0 . Put $R_{j}=R_{\left(P_{R}\left(M_{j}\right)\right.}$ and $P_{j}=P_{R}\left(M_{j}\right)_{\left(P_{R}\left(M_{j}\right)\right.}$ for simplicity. By Theorem 3.6, $M_{j}$ is isomorphic to $E_{R_{j}}\left(\overline{R_{j} / P_{j}}\right)$. Notice that any two different ideals $P_{R}\left(M_{j}\right)$ determine non-isomorphic modules $M_{j}$. Let $p_{j}$ denote a generator of $P_{j}, P_{j}=p_{j} R$. If $\beta_{j}$ is infinite, then $M\left[p_{j}\right]$ is of infinite length over $Z_{R}(M)$, which contradicts pseudo-strong minimality. Similarly, if $r \in R$ is in infinitely many $P_{j}$ 's, then $M[r]$ is of infinite length over $Z_{R}(M)$ and again contradicts the p. s. m. assumption.

Conversely, suppose that $M$ satisfies 1 or 2 . When $R$ is a skew field (and 2 holds), it is easily seen that $M$ is p. s. m. as claimed. So assume 1 . Then $M$ is of infinite length over $R$ : this is obvious if $\beta_{P}>0$ for some $P$, and follows from the assumption on $Q(R)$ and $\alpha$ otherwise. Also, every pp-subgroup of $M$ is of finite length over $Z_{R}(M)$. In fact, as $R$ is a right uniserial domain, it suffices to check this condition for annihilators $M[r]$ with $r \in R$, and in this reduced framework the claim follows because $Q(R)$ is torsionfree, every $r \in R$ lies in the direct summand corresponding to an ideal $P$ for at most finitely many $P$, and, for any $P, \beta_{P}$ is finite and the ideals of $R_{(P)}$ are as described. $\dashv$

\section{Weak minimality}

We deal here with weak minimality over serial rings. Accordingly we fix a serial ring $R$ and a complete set of pairwise orthogonal indecomposable idempotents $e_{0}, \ldots, e_{n}$ in $R$. Recall that a module $M$ is called weakly minimal (w. m.) if and only if $M$ is infinite and every pp-subgroup of $M$ is either finite or of finite index. Actually this is just the translation in the context of modules of the general definition of weak minimality, according to which w. m. structures are just those of $U$-rank 1 . In any case, over a serial ring $R$ weak minimality can be characterized in a simpler way, only referring to divisibility and torsion conditions.

Lemma 4.1 Let $M$ be a module over a serial ring $R$. Then $M$ is w. $m$. if and only if, for every $r \in R$, either $M r$ is finite or $M r$ is of finite index in $M$ and $M[r]$ is finite.

Observe that the first case "Mr is finite" implicitly implies that $M[r]$, i. e. the kernel of the right multiplication by $r$ in $M$, is of finite index.

Proof. The direction from left to right is true in general (see [9], $₹ 7$ ). Conversely, take a pp-formula $\phi(v)$ over $R$. Due to [1], Corollary 1.6 (see also [17], Lemma 11.1), $\phi(M)$ coincides with a finite sum of pp-subgroups $M s \cap M[r]$ with $s \in e_{i} R, r \in R e_{j}, i, j \leq n$. Accordingly write $\phi(M)=\sum_{k<m}\left(M s_{k} \cap M\left[r_{k}\right]\right)$. If both $M s_{k}$ and $M\left[r_{k}\right]$ have finite index for some $k \leq m$, then the same is true of $\phi(M)$. Otherwise for every $k \leq m$ either $M s_{k}$ or $M\left[r_{k}\right]$-and consequently $M s_{k} \cap M\left[r_{k}\right]-$ is finite, whence $\phi(M)$ is finite, too. $\dashv$

Observe once again that a w. m. module may not satisfy $(\star)$. But let us illustrate why, even in the w. m. case, our analysis can be reduced from serial 
to uniserial rings. Take a module $M$ over the serial ring $R$. Then $M$ decomposes as $\oplus_{i \leq n} M e_{i}$ where, for every $i \leq n, M e_{i}$ is an abelian group and even a module over the uniserial ring $e_{i} R e_{i}$, but not necessarily a module over $R$ (although $M e_{i}$ is a pp-subgroup of $M$ over $\mathrm{R}$ ). Hence $M$ is to be regarded here as an abelian group but not as a module over $R$. It is easy to see what follows.

Lemma 4.2 Let $M$ be a w. m. module over a serial ring $R$. Then there is a unique $i \leq n$ for which $M e_{i}$ is of finite index (and, for $j \neq i, M e_{j}$ is finite).

Proof. As $M$ decomposes as $\oplus_{i \leq n} M e_{i}$ as an abelian group and $M$ is infinite, there is some $i \leq n$ such that $M e_{i}$ is infinite, and hence of finite index in $M$. But, if for $i<j \leq n$ both $M e_{i}$ and $M e_{j}$ are of finite index, then also $\left(M e_{i}\right) e_{j}=M e_{i} e_{j}=M 0=0$ is, whence $M$ is finite. $\dashv$

Assume for simplicity that $i=0$, so $M e_{0}$ is of finite index, while $M e_{i}$ is finite when $i>0$. Thus, in order to describe the structure of the w. m. $R$-module $M$, both

1. $M e_{0}$ as a module over the uniserial ring $e_{0} R e_{0}$, and

2. $\oplus_{0<i \leq n} M e_{i}$ as a finite module over $\sum_{0<i, j \leq n} e_{i} R e_{j}$

have to be considered. But this is not enough, and there is a third point to be contemplated, i. e.

3. for every $r \in R$ and $i>0$, two group morphisms, the former from $M e_{0}$ into $M e_{i}$ sending any $m e_{0}$ into $m e_{0} r e_{i}$, the latter from $M e_{i}$ into $M e_{0}$ taking any $m e_{i}$ to $m e_{i} r e_{0}$.

These morphisms contribute to the clarification of the $R$-module structure of $M$. Observe that the kernel of the former morphism has finite index in $M e_{0}$ because $M e_{i}$ is finite, and for the same reason the image of the latter morphism is also finite. One might think that one would have to consider further morphisms, such as, for $0<i<j \leq n$ and $r_{i}, r_{j} \in R$,

$$
m e_{0} \rightarrow m e_{0} r_{i} e_{i}+m e_{0} r_{j} e_{j}
$$

and

$$
m_{i} e_{i}+m_{j} e_{j} \rightarrow m_{i} e_{i} r_{i} e_{0}+m_{j} e_{j} r_{j} e_{0},
$$

and so on; but note that the kernel of the former morphism is just the intersection of the kernels of multiplications by $r_{i} e_{i}$ and $r_{j} e_{j}$, and similarly the image of the latter morphism if the sum of the images of $r_{i} e_{0}$ and $r_{j} e_{0}$.

Anyway the main ingredient in this description of $M$ is $M e_{0}$ as a module over $e_{0} R e_{0}$. Notably $M e_{0}$ is w. m. over $e_{0} R e_{0}$. To see this, note that every ppformula $\phi(v)$ over $e_{0} R e_{0}$ generates by relativization to $v e_{0}=v$ a pp-formula $\phi^{\prime}(v)$ over $R$ such that $\phi^{\prime}(M)=\phi\left(M e_{0}\right)$; hence $\phi\left(M e_{0}\right)$ is either finite or of finite index in $M e_{0}$ because the same is true of $\phi^{\prime}(M)$ in $M$. But the relationship between $M$ and $M e_{0}$ is even stronger, and the following holds. 
Proposition 4.3 Let $M$ be a module over the serial ring $R$, assume that $M e_{0}$ is of finite index and that $M e_{i}$ is finite for $0<i \leq n$. Then $M$ is $w . m$. over $R$ if and only if $M e_{0}$ is w. m. over $e_{0} R e_{0}$.

Proof. The direction from left to right has just been proved. Conversely suppose that $M e_{0}$ is w. m. over $e_{0} R e_{0}$. Take any $r \in R, r=\sum_{j \leq n} r e_{j}=\sum_{i, j \leq n} e_{i} r e_{j}$. We have to show that either $M r$ is finite or $M r$ is of finite index and $M[r]$ is finite. We know that either $M e_{0} r e_{0}$ is finite or $M e_{0} r e_{0}$ is of finite index and $M e_{0}\left[e_{0} r e_{0}\right]$ is finite. Observe

$$
M r=\sum_{i, j \leq n} M e_{i} r e_{j}=M e_{0} r e_{0}+\sum_{i>0 \text { or } j>0} M e_{i} r e_{j}
$$

where the latter summand is finite because each $M e_{h}$ is for $h>0$. Thus, if $M e_{0} r e_{0}$ is finite, then $M r$ is, which yields the first case in Lemma 4.1. In the same way, if $M e_{0} r e_{0}$ is of finite index in $M e_{0}$, then $M r$ is of finite index in $M$, because $M e_{0}$ has finite index in $M$, hence [ $\left.M: M e_{0} r e_{0}\right]$ is finite and, as $M e_{h}$ is finite for $h>0,[M: M r]$ is finite, too. So, in order to get the second case in Lemma 4.1, we have to show that $M[r]$ is finite if $M e_{0}\left[e_{0} r e_{0}\right]$ is. Note that, for $m \in M, m r=\sum_{j \leq n} m r e_{j}$ is 0 if and only if $m_{r e} e_{j}=0$ for every $j \leq n$. For $j>0,\left\{m \in M: m_{r} e_{j}=0\right\}$ has finite index in $M$ because the group morphism $m \rightarrow m r e_{j}$ has a finite image. So consider $j=0$. Every element $m \in M$ such that $m r e_{0}=0$, equivalently $m e_{0} r e_{0}=-\sum_{0<i \leq n} m e_{i} r e_{0}$, also satisfies $m e_{0} r e_{0} \in \sum_{0<i \leq n} M e_{i} r e_{0}$ where $\sum_{0<i \leq n} M e_{i} r e_{0}$ is finite. Consequently $\{m \in$ $\left.M: m r e_{0}=0\right\}$ is finite provided that $M\left[e_{0} r e_{0}\right]$ is. Write $m=\sum_{i \leq n} m e_{i}$ and notice that $m e_{0} r e_{0}=0$ if and only if $\left(m e_{0}\right) e_{0} r e_{0}=0$, where $m-m e_{0}=$ $\sum_{0<i<n} m e_{i}$ can assume only finitely many values. Thus $M\left[e_{0} r e_{0}\right]$ is finite when $M e_{0}\left[e_{0} r e_{0}\right]$ is. $\dashv$

Hence Proposition 4.3 again restricts the weak minimality analysis from serial to uniserial rings. So let $R$ be uniserial, and let $M$ be a w. m. module over $R$. Up to elementary equivalence, we can assume that $M$ is a direct sum of indecomposable pure injective modules, $M=\oplus_{h} M_{h}$. For a w. m. $M$, each summand $M_{h}$ is either finite or w. m. as well. As observed in [17], Corollary 11.5 (referring in its turn to [1]), each w. m. summand $M_{h}$ is uniserial over its endomorphism ring and consequently pp-uniserial. In other words the ppsubgroups of $M_{h}$ reduce to those of the forms $M_{h} s$ or $M_{h}[r]$ with $r$ and $s$ in $R$, and are linearly ordered by inclusion. Moreover weak minimality implies that the order type of this linear order is $\omega+\omega^{\star}$ in the worst case, corresponding to countably many finite pp-subgroups (ordered as $\omega$ ) and then countably many pp-subgroups of finite index (ordered as the reverse order $\omega^{\star}$ ).

Further information and details can be obtained specifying $M$ or $R$. For instance, assume that $R$ is an integral domain and that $M$ is divisible, hence $M s=M$ for every nonzero $s \in R$. This means that $M_{h}[s]$ is finite for every $s$, whence the lattice of pp-subgroups of $M_{h}$ is either finite or linearly ordered of type $\omega+1$. Conversely, if $M$ is $R$-torsionfree, i. e. $M[r]=0$ for every nonzero $r \in R$, then for all $h M_{h} r$ has finite index in $M_{h}$ and the lattice of pp-subgroups 
of $M_{h}$ is either finite or linearly ordered of type $1+\omega^{\star}$. On the other hand, a complete classification of $\mathrm{w}$. m. modules over a commutative valuation domain is given in [6].

As a further example let us consider the serial, right but not left noetherian ring

$$
R=\left(\begin{array}{cc}
\mathbf{Z}_{(p)} & \mathbf{Q} \\
0 & \mathbf{Q}
\end{array}\right)
$$

where $p$ is a prime and $\mathbf{Z}_{(p)}$ denotes the localization of integers at $p$ (see [17], 11.16). Then

$$
e_{1}=\left(\begin{array}{ll}
1 & 0 \\
0 & 0
\end{array}\right), \quad e_{2}=\left(\begin{array}{ll}
0 & 0 \\
0 & 1
\end{array}\right)
$$

are a complete system of pairwise orthogonal indecomposable idempotents in $R$. Up to elementary equivalence we can single out the w. m. $R$-modules among the direct sums of indecomposable pure injective $R$-modules. They are listed in detail in [17], 11.16, so let us refer to their description there.

(i) $\mathbf{Z} / p^{n}(n \geq 1), \mathbf{Z} / p^{\infty}, \widehat{\mathbf{Z}_{p}}$ and $\mathbf{Q}$ can be viewed as $R$-modules provided we agree that $e_{1}$ acts as 1 and $e_{2}$ (and $\left(\begin{array}{ll}0 & 1 \\ 0 & 0\end{array}\right)$ as 0 . All of them are indecomposable pure injective, and either finite or weakly minimal.

(ii) $\mathbf{Q}$ can be regarded as an (indecomposable pure injective) $R$-module even with respect to an identical action of $e_{2}$ and a zero action of both $e_{1}$ and $\left(\begin{array}{ll}0 & 1 \\ 0 & 0\end{array}\right)$; as such it is again weakly minimal.

The further indecomposable pure injective $R$-modules (the injective hull and the pure injective hull of $e_{1} R$, respectively) are not w. m. because $\left(e_{1} R\right)\left[e_{1}\right]$ -the pp-subgroup of the elements in $e_{1} R$ annihilated by $e_{1}$ - is both infinite and of infinite index in $R$, and then in their hulls.

In order to build new w. m. $R$-modules $M$ in this framework, i. e. among direct sums of indecomposable pure injective $R$-modules, one can either

- form in (i) a finite direct power of either $\mathbf{Z} / p^{\infty}$ or $\widehat{\mathbf{Z}_{p}}$ and possibly add finitely many direct summands among the $\mathbf{Z} / p^{n}$ 's $(n \geq 1)$ and arbitrarily many direct summands isomorphic to $\mathbf{Q}$, or

- take in (i) or (ii) as many copies of $\mathbf{Q}$ as one wants, and possibly add a finite direct summand from (i).

Note that in both cases either $M e_{1}$ or $M e_{2}$ has finite index in $M$.

\section{References}

[1] P. Eklof - I. Herzog, Model theory of modules over a serial ring, Ann. Pure Appl. Math. 72 (1995), 145-176 
[2] A. Facchini, Module theory, Birkhäuser, 1998

[3] I. Herzog - V. Puninskaya, The model theory of divisible modules over a domain, Fund. Math. Appl. 2 (1996), 563-594

[4] I. Herzog - P. Rothmaler, Modules with regular generic types I, II, III, in: A. Nesin - A. Pillay (editors), The model theory of groups, Notre Dame Mathematical Lectures 11, University Press, Notre Dame, 1989, 138-176

[5] C. Jensen - H. Lenzing, Model theoretic algebra, with particular emphasis on fields, rings and modules, Gordon and Breach, 1989

[6] S. Leonesi - S. L'Innocente - C. Toffalori, Weakly minimal modules over integral group rings and over related classes of rings, Math. Log. Quart. 51 (2005), 613-625

[7] S. L'Innocente - V. Puninskaya - C. Toffalori, Strongly minimal modules over group rings, Comm. Algebra 33 (2005), 2089-2107

[8] S. L'Innocente - V. Puninskaya - C. Toffalori, Minimalities and modules over Dedekind-like rings, Comm. Algebra, 34 (2006), 2453-2466

[9] J. Loveys, On locally modular weakly minimal theories, Arch. Math. Logic 32 (1983), 173-194

[10] J. Loveys, Weakly minimal groups of unbounded exponent, J. Symbolic Logic 55 (1990), 928-937

[11] J.C. McConnell - J.C. Robson, Noncommutative Noetherian rings, American Mathematical Society, 2001

[12] B. Müller, The structure of serial rings, pp. 249-270 in: Methods in Module Theory, Marcel Dekker, 1992

[13] M. Prest, Model theory and modules, Cambridge UP, 1988

[14] V. Puninskaya, Strongly minimal modules over right distributive rings, Algebra and Logic 35 (1996), 345-358

[15] V. Puninskaya, Strongly minimal modules, J. Math. Sci. 114 (2003), 11271157

[16] V. Puninskaya, The model theory of divisible modules over Ore domains, unpublished notes

[17] G. Puninski, Serial rings, Kluwer, 2001 
[18] G. Puninski - R. Wisbauer, $\Sigma$-injective modules over left duo and left distributive rings, J. Pure Applied Algebra 113 (1996), 55-66

[19] M. Ziegler, Model theory of modules, Ann. Pure Appl. Logic 26 (1984), $149-213$ 